\title{
The impact of age and apoE genotype on the fasting and postprandial TAG response
}

\author{
A. L. Carvalho-Wells ${ }^{1}$, G. Gill-Garrison ${ }^{2}$, E. Olano-Martin ${ }^{1}$, K. G. Jackson ${ }^{1}$ and A. M. Minihane ${ }^{1}$ \\ ${ }^{1}$ Department of Food Biosciences, University of Reading, Reading RG6 6AP, UK and ${ }^{2}$ Sciona Inc, Boulder, CO 80302, USA
}

Carriers of the apoE4 allele have been shown to have increased risk of CVD by as much as $40-50 \%{ }^{(1)}$. Traditionally this increased risk has been attributable to higher total cholesterol (TC) and LDL-cholesterol (LDL-C) levels. However, the modest increases in TC and LDL-C observed in apoE4 carriers relative to the common apoE3 genotype cannot fully explain the greater CVD risk in this subgroup. The current study examined the impact of apoE genotype on the fasting and postprandial TAG response.

Healthy men ( $n$ 145) and women ( $n$ 109) with a mean age of 53 (SE 1.0) years, BMI of 26.4 (SE 0.2$) \mathrm{kg} / \mathrm{m}^{2}$ ) and fasted triacylglycerol (TAG) of $1.63 \mathrm{mmol} / \mathrm{l}$ (SE 0.05) underwent a postprandial assessment. After consumption of a standard breakfast (time $0 \mathrm{~min}, 49 \mathrm{~g}$ fat) and lunch (time $330 \mathrm{~min}, 29 \mathrm{~g}$ fat), blood samples were taken at regular intervals until $8 \mathrm{~h}$ post-breakfast for analysis of fasting lipids and postprandial TAG using automated colorimetric assays. Genotype groups were arranged as E2 carriers (E2/E2 and E2/E3, $n$ 47), E4 carriers (E4/E4 and E4/E3, $n$ 66) and homozygous E3/E3 ( $n$ 142).

Fasting TAG was shown to be $17 \%$ and $26 \%$ higher in E2 and E4 carriers relative to the E3/E3 genotype $(P=0.001)$. This trend was also evident in the postprandial TAG response with a $13 \%$ and $22 \%$ higher area under the curve (AUC) in E2 and E4 carriers $(P=0.038$ ). As age has been shown to influence the magnitude of the postprandial TAG response, the subjects were subdivided into a younger ( $\leq 50$ years) and older ( $\geq 51$ years) group. A significant impact of apoE genotype on both fasted TAG $(P=0.053)$ and postprandial TAG AUC $(P=0.028)$ was observed in the older group with higher levels in E4 carriers relative to the wild-type E3/E3 group. However, this relationship was not evident in the younger cohort ( $\leq 50$ years).

A

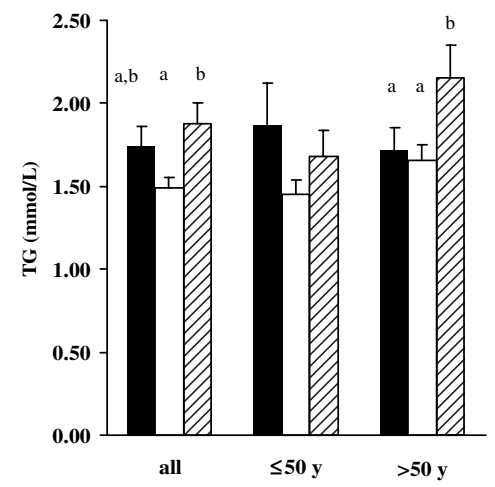

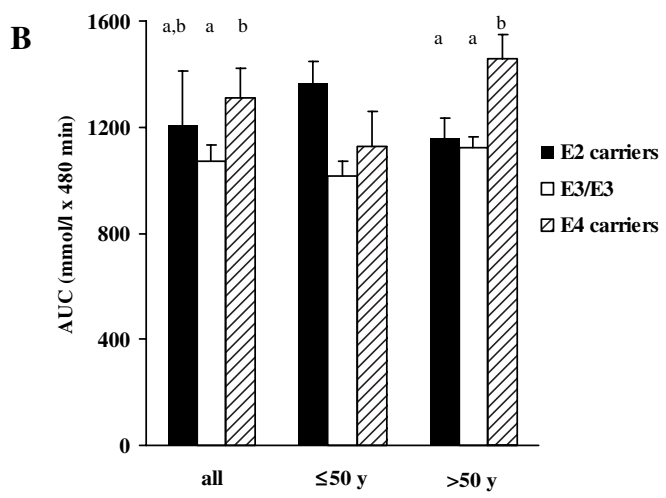

Figure. Fasting TAG levels (A) and AUC for the postprandial TAG response (B) in the three genotype groups. Data are presented for the group as a whole and for the two age-groups ( $\leq 50$ years and $>50$ years). ${ }^{\mathrm{a}, \mathrm{b}}$ Means with unlike superscript letters were significantly different between the genotype groups $(P<0.05)$.

The data suggest that age partly determines the impact of apoE genotype on TAG concentrations. The molecular basis for higher TAG in E4 carriers is unknown, but may be in part a result of the reported lower recycling of internalised apoE to the hepatocyte surface ${ }^{(2)}$, which would lower the concentration of apoE ligand available for TAG-rich lipoprotein internalisation by the liver. Further investigations in to molecular mechanisms are warranted.

1. Minihane AM, Jofre-Monseny L, Olano-Martin E et al. (2007) Proc Nutr Soc 66, 183-197.

2. Heeren J, Grewal T, Laatsch A et al. (2004) J. Biol. Chem 279 (53), 55483-55492. 